\title{
INTRODUCTION OF ULTRA-PRECISION TECH SHOP
}

\author{
Geonhee Kim ${ }^{1,+, *}$, Kyesung Lee ${ }^{1}$, Hwan Hur ${ }^{1}$, Sangwon Hyun ${ }^{1}$, Hwanjin Choi ${ }^{1}$, Minwoo Jeon ${ }^{1}$ \\ ${ }^{1}$ Korea Basic Science Institute, Gwahak-ro, Yuseng-gu, Daejeon, Korea \\ +*Presenting and Corresponding Author: kgh@kbsi.re.kr
}

\begin{abstract}
The world's optical parts market is entering a phase of free-form optics beyond existing aspherical optics. Demand for ultraprecision optical system is exploding in high-tech industries such as astronomical telescopes, high-powered lasers, satellites, unmanned equipment, wearable devices, and automobiles. To meet this demand, the Korea Basic Science Institute (KBSI) has opened a Ultra Precision Tech Shop (UPTS), a world-class precision processing facility. The UPTS utilizes KBSI equipment to carry out technical support for the fabricating sector and the manufacture of high-performance optical components and modules. UPTS also contributes to securing competitiveness of the manufacturing industries by providing a high-quality machining \& measuring equipment, and the provision of services that are difficult to obtain and use. Further, it plans to construct a joint research network with the world's leading research institutes to improve the credibility of the domestic optical industry.
\end{abstract}

KEYWORDS: Magneto-Rheological Finishing, Diamond Turning Machine, Interferometer, Atomic Force Microscope

\section{INTRODUCTION}

As our society continues develop, there are increasing demands for developing and using high-value added machines for research. In particular, advanced countries lead the field of ultra-precision technology and machines, and Korea imports most machines for ultra-precision manufacturing process. In this circumstance, the Division of Scientific Instrumentation of Korea Basic Science Institute runs the Ultra-precision Tech shop to develop key components and build an ultra-precision research center.

\section{ULTRA-PRECISION MANUFACTURING TECHNOLOGIES AND PROCESS}

Ultra-precision freeform machining which is an exemplary technology of UPTS is used to finish and evaluate various forms of optical surfaces, for example, off-axis aspheric, cylindrical, toroidal, and MLA surfaces as well as finishing typical spheric and aspheric optical surfaces at a precision not greater than several $\mathrm{nm}$. From the drawing and design of a final product, the ultra-precision fabrication process starts with a preliminary machining or grind. Next, a coordinate measuring machine is used to carry out 3D shape measurement and evaluation to achieve improved precision in next steps. Ultra-precision fabrication comprises the steps of precision machining and polishing depending on materials, and more precise finishing is implemented through magneto-rheological surface correction and finishing. A finished product is made after the final step of shape measurement and evaluation.

\section{EQUIPMENT ON THE UPTS}

The machines required in the ultra-precision optical engineering process include ultra-precision machining, polishing, measurement and evaluation instruments. First, the single-point diamond turning machine, one of the largest ultra-precision machining instruments in Korea, can fabricate 3-dimensional freeform surfaces in nm level of precision by means of the simultaneous control of multi-axis and fast tool servo system. The aspheric polishing machine can precisely polish aspheric and free-form glass surfaces through a physical and chemical polishing technique. Last, the MRF polishing and finishing machine corrects free-form surfaces of various materials through a magneto-rheological polishing process to achieve extremely high performance in terms of surface roughness and form accuracy. A representative measurement and evaluation instrument is the Ultra-precision atomic force 3D Profiler, which contacts its diamond probe to a measured surface under micro pressure to measure the shape of 3D surfaces while moving it. Moreover, another latest measuring instrument is the Subaperture Stitching Interferometer, which logically divides the whole optical surface into several tens of smaller sub- 
apertures measures them with a laser interferometer and stitching them one by one to recover the full-aperture 3D aspheric shape. The non-contact interferometric 3D Profiler obtains and analyzes the 3D shape and surface roughness of local area of measuring surfaces.

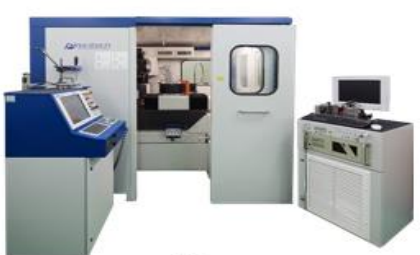

(a)

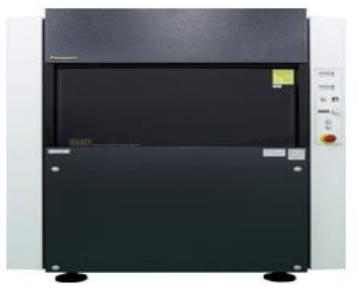

(c)

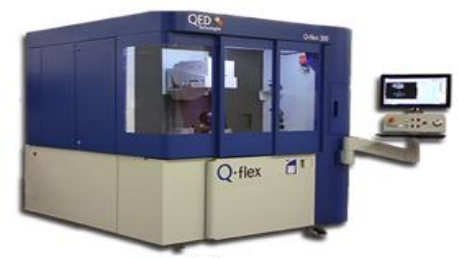

(b)

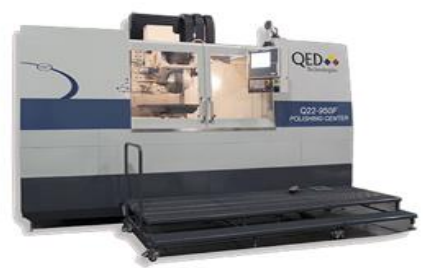

(d)

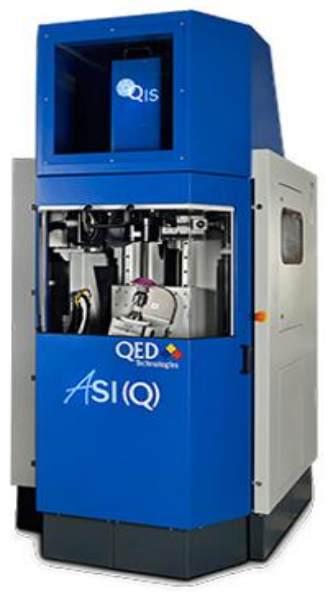

(e)

Figure 1 (a) SPDTM (b) MRF (c) Atomic force surface profiler (d) Large-aperture MRF (e) Stitching interferometer

\section{APPLICATION OF ULTRA-PRECISION MANUFACTURING TECHNOLOGIES}

The materials fabricated by means of the ultra-precision system have been used in various fields, for example, IT, NT and ST. We will continue to develop next-generation key optical components by building a global hub for developing optical analytic instruments to contribute to making Korea more competitive in the global market. UPTS of Korea Basic Science Institute makes every effort to develop optical analytic instruments and build an open convergence system which is required in the future. It will contribute to creating new industries of high added values in various fields, for example, high-energy laser optical components, optical devices used in the air and space industry, and key optical modules used in self-driving cars.
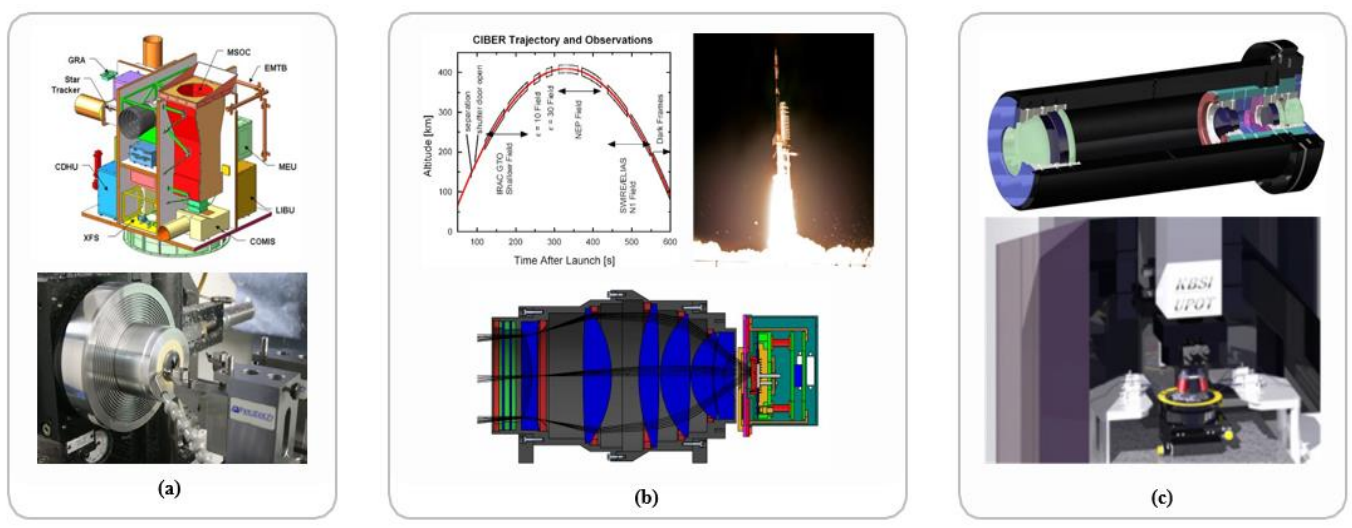

Figure 2 (a) MIRIS optical payload (b) CIBER infrared optics (c) Thermal imaging microscope

\section{FUTURE WORKS}

UPTS looks at the wider world and into the future far ahead. It has built a ground including human resources, space and machines and constructed a cooperative network in Korea and other countries to provide more support for the industry, universities and research institutes. This aims to enhance technology and service of UPTS, be a leader in construction of the ecosystem for the independent machine industry for research in Korea, and a representative institution in the global community. 